\title{
THE EXTENDED CLUSTERING AD HOC ROUTING PROTOCOL (ECRP)
}

\author{
${ }^{1}$ Buthayna Al-Sharaa \\ ${ }^{1}$ Department of Electrical Engineering, Al Balqa Applied University, Jordan \\ buthayna74@hotmail.com
}

\begin{abstract}
Ad hoc networks are a collection of mobile nodes communicating via wireless channels without any fixed infrastructure. Because of their ease and low cost of building, ad hoc networks have a lot of attractive applications in different fields. The topology of ad hoc networks changes dynamically, and each node in the network can act as a host or router. With the increase in the number of wireless devices and large amount of traffic to be exchanged, the demand for scalable routing protocols has increased. This paper presents a scalable routing protocol, based on AODV protocol, called the Extended Clustering Ad Hoc Routing Protocol (ECRP). This is a hybrid protocol, which combines reactive and proactive approaches in routing. The protocol uses the Global Positioning System to determine the position of certain nodes in the network. The evaluation methodology and simulation results obtained show that the protocol is efficient and scales well in large networks.
\end{abstract}

\section{Keywords}

Ad hoc networks, routing protocols, hybrid protocols.

\section{INTRODUCTION}

Ad hoc network is a set of wireless mobile nodes connected via wireless channels. Ad hoc networks form a dynamic network with no fixed infrastructure. Nodes in the network use limited range radio signals to transmit data. Nodes that are unable to communicate directly use intermediate nodes to forward packets for them [1]. Since ad hoc networks do not require any infrastructure, they are relatively not expensive. Ad hoc networks can be applied in many areas where natural conditions or time matters make it impossible to have a structured network. Such applications can be found in emergency services, battlefields, conference rooms, homes, and others.

Ad hoc networks have a lot of challenges. One of these challenges is the dynamic change in topologies which lead to route changes. The system or application will not be aware of these changes until it tries to use one of the routes which have been broken. Also network nodes usually suffer from short battery lifetime and limited capacities. The bandwidth in wireless networks is limited. Some factors which exist in ad hoc networks consume the bandwidth, some of these factors are: the broadcast nature of the communications, packet loss due to transmission errors and frequent disconnections [2].

Each node in the network can act as a router and as a host[3]. The function of a routing protocol in ad hoc network is to establish routes between different nodes. Many protocols have been proposed. In general, ad hoc routing protocol can generally be classified into two main classes: Table driven(proactive) and On-Demand (reactive) protocols. In addition to the proactive and 
International Journal of Computer Networks \& Communications (IJCNC) Vol.5, No.3, May 2013

reactive routing protocols, other categories of ad hoc routing protocols exist. These categories are: hybrid (Pro-Active/Reactive), hierarchical, geographical, power aware, multicast, geographical multicast (Geocasting), and etc. Different numbers of ad hoc protocols can be found in each of these categories.

Hierarchical routing protocols can be used to solve the problem of user and traffic increase. These protocols tend to organize nodes in a hierarchical manner. Another characteristic of these routing protocols is that the number of nodes involved in the search for a destination is reduced. Using hierarchical protocols enhance the performance of the ad hoc networks in several ways. First, better utilization of node resources such as memory and power is made. Secondly, better bandwidth utilization is achieved. Finally, secure communications can be provided.

\section{Related Work}

Zone Routing Protocol (ZRP) [4] combines proactive and reactive approaches. In ZRP, the network is divided into zones. This routing protocol uses routing schemas:Intra-zone routing and Inter-zone routing. Intra-zone routing uses the proactive method to maintain routes to all nodes within the source node's own zone. Inter-zone routing uses the on-demand approach to determine routes to nodes outside a zone.

Virtual Backbone Routing (VBR) [5] is a scalable hybrid routing framework for ad hoc networks, which combines local proactive and global reactive routing components over a variable-sized zone hierarchy. It is based on the ZRP's concept of combining proactive local zone routing with reactive global route queries. However, unlike ZRP, VBR uses the notion of a virtual backbone (VB) to efficiently direct the route querying control traffic. The reactive component of VBR restricts the route queries within the virtual backbone only, thus improving the overall routing efficiency.

The Adaptive Routing using Clusters(ARC) is a hierarchal routing protocol [6]. ARC is based on LCA protocol. The main differences between ARC and LCA are that ARC supports the use of multiple gateways between clusters, whereas LCA defines that only one cluster must be selected between a given set of clusters. Also the two protocols use two different cluster formation techniques. LCA uses node identifier to select cluster leaders, whereas ARC uses connectivity. In addition LCA requires that nodes must remain synchronized and must maintain an accurate global time so that the control channel may be divided into epochs.

In this paper, I present the Extended Clustering Routing Protocol (ECRP). It is a scalable routing protocol for ad hoc networks. Simulation result shows that comparing with AODV, ECRP scales well to large network, and has low overhead and reasonable end-to-end delay. The rest of the paper is organized as follows. In Section III, I present the Extended Clustering Routing Protocol. Section IV describes the simulation environment and presents the simulation results. Section V presents the conclusion.

\section{Protocol Description}

The ECRP is a hybrid routing protocol. The protocol divides the rounting process mainly into two classes: cluster-based routing and network -based routing. Initially, a node starts by itself sending out hello messages to explore its neighbors. The TTL of each hello message is 1 or 2 hop. This operation is repeated in different areas of the network. The protocol has two different modes: reactive and proactive. When the source-destination connection is global, the protocol operates in the reactive mode. on the other hand, when the source-destination connection is local the proactive mode is used. 
International Journal of Computer Networks \& Communications (IJCNC) Vol.5, No.3, May 2013

In general each cluster contains a base, a backup node, and regular nodes. The regular nodes within a cluster have work in common and the connections among them are the dominant type of connections in the network. The base is a central point used to coordinate the operations within the cluster and accomplish the external connections. Each base has an ID number. The base ID number will be used also to identify the cluster. The backup node recives from the base all the control messages and learns all the routes discovered so far. The existence of the backup node enhances the network performance since it replaces the base when it's off work. The backup node is usually placed in the area between two bases. When any base is down for some reason, it takes its place. Putting the backup node in the path of external routes decreases the control tarffic.The TTL of each message sent by all nodes within a cluster is 2 hop. All nodes within the cluster exchange hello messages. The base node contains a neighbor table. This table is updated periodically by the regular nodes within the cluster.

In the network, there are certain small areas where a base node can exist. These areas are called the control points. Each node holds a map of these points. The method used by nodes to learn their coordinates in the network is the Global Positioning System GPS $[7,8]$ or we can use any other type of positioning service [9]. Each control point should be occupied by a base and should not be left empty. In this way, all clusters of network can be reached.

Regular nodes are allowed to exchange data messages only. Whenever a regular node receives a control message it immediatelly discards it. The different control messages used by the protocol are: route request (RREQ), route reply (RREP), route reply acknowledgments (RREP_ACK) and route error (RERR). The control messages are only used by bases to build routes and maintain them.

\section{1. Cluster-Based Routing}

Cluster-based routing is used by regular nodes to communicate within a cell. Regular nodes don't know if the destination is a neighbor or not. Nodes simply broadcast their data messages. The TTL of these messages is 2 hops. The sent message has two situations. The first is that it is received by the destination, which is in this case a neighbor. The base node in cluster-based routing is not involved. The neighbor table is used by the base to determine the set of regular nodes in its cluster. The other situation is that the destination is an external node. Here, only the base will accept this message and execute a network-based routing.

\subsection{NETWORK-BASED ROUTING IN ECRP}

This routing tequniche is used if the destination is a global node, here two cases exit. The first case is that the base contains a route to destination, this route is used to transmit the message. The other case is that the base does not have a route to destination, the base here should initiate a route discovery phase. The RREQ messages are used to discover new routes. These messages are only processed by bases and the bridging backup nodes in the network. Local nodes discard RREQ. By this, the control overhead is reduced and network bandwidth is preserved. All the routing messages are passed to the backup nodes which will eventually hold the discovered routes. Before a base initiates a route discovery phase, it increases the TTL of the message. Figure1 below outlines the procedure for the ECRP algorithm. 


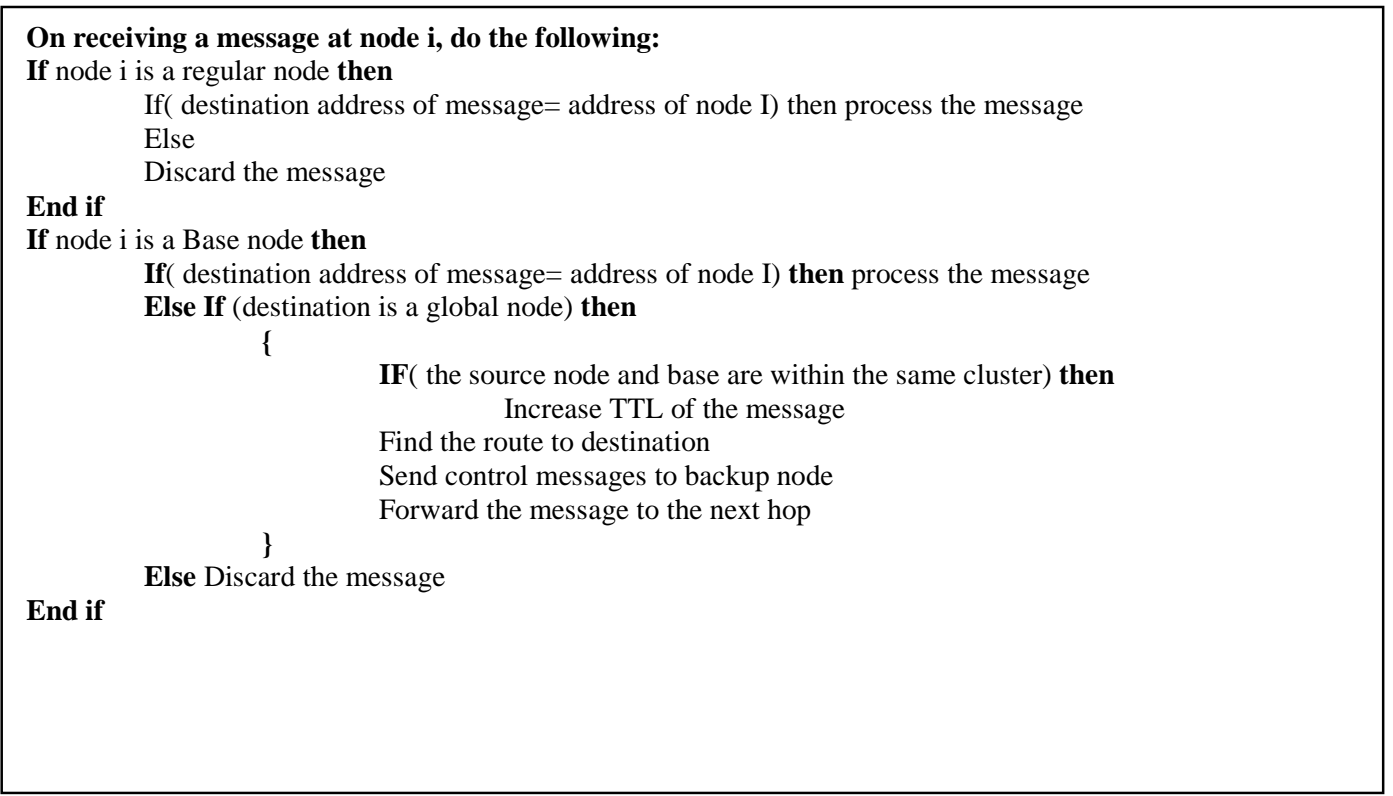

Figure1. The ECRP Procedure

\section{PERFORMANCE ANALYSIS}

\subsection{Simulation Environment}

We evaluated the performance of ECRP by comparing ECRB with Ad-hoc On-Demand Distance Vector (AODV) routing protocol [10]. We implemented ECRP using ns2 simulator [11] and the wireless extensions developed by CMU. NS2 can run under Linux and windows. But in our work the environment chosen was Linux. NS2 contains the implementation of a number of protocols; AODV is one of them. The characteristics of radio model are a shared-media radio with $2 \mathrm{Mb} / \mathrm{sec}$ transmission rate and radio range of 250 meters. The wireless networking extensions made by CMU's Monarch project which are added to NS2 [12] are used.

Before a mobile node can be implemented in any simulation it needs to be configured. The configuration of a mobile node includes specifying its addressing type, the interface queue, the physical type and the ad hoc routing protocol, etc. The MAC layer is the IEEE 802.11 MAC protocol with Distributed Coordination Function (DCF) which uses Request-to-send (RTS) and Clear-to-send (CTS) control frames for unicast packet. The Carrier Sense Multiple Access/Collision Avoidance (CSMA/CA) is the access frame [13].

\subsection{Traffic and Mobility Patterns}

The application setdest in the NS2 simulator is used to generate the mobility model. The traffic sources use the random waypoint algorithm. The difference between every movement file is the pause time of nodes. Pause times are varied from 0 to 200 seconds with a step of 20 . In these files each node stops for a predetermined pause time, after that it starts moving toward a random destination. The highest system mobility is when the pause time equals 0. At pause time 200 seconds no movement occurs. The speeds of nodes vary in a range from 0 to $10 \mathrm{~m} / \mathrm{sec}$. The source and destination nodes are randomly selected. The cbrgen.tcl script in ns2 simulator is used for traffic generation. The type of traffic chosen is Constant Bit Rate (CBR) flowing over UDP. The 
connections between nodes were chosen randomly. The connections established contain local and global ones. Each CBR session transmits 1000 packets of 512 bytes at a rate of 4 packets/sec. The packet rate chosen does not overflow the interface queue ensuring that packet losses will only be due to the wireless channel. In order to test the effect of traffic load, the number of connections between nodes is varied from 10 to 50 connections. Identical traffic and mobility scenarios are used for both routing protocols. For AODV, we used the original code in the ns2 package.

\subsection{Results and Discussion}

1) Packet Delivery Fraction PDF: this is the ratio of the data packets received by the destinations to those that were generated by the sources. Figure1 and figure 2 show the packet delivery fraction of AODV and ECRP under different traffic load. In this system, the total traffic contains interand intra - cluster connections. As figure1 shows, with law pause time the PDF of ECRP and AODV are low. At low pause times, mobility is higher, and routes break more, which lead to low fractions of packet delivery. The reason why the PDF of ECRP is higher than that of AODV is that in ECRP the connections are mostly one hop long. Also, all external routes pass through the bases and backup nodes. As we specified in this paper, these nodes are almost fixed, so the routes are stable. Figure 2 shows that as the traffic load increase, the PDF of both ECRP and AODV decrease. The PDF of ECRP at high traffic load is better than that of AODV. The reason for this is that most of the connections are made inside the cell. In ECRP the path length of packets inside a cell is one hop, and the source directly transmits the packets to the destination. On the other hand, in ADOV if a node needs to communicate with a destination it will directly broadcast a RREQ whether this destination is a neighbor or not. The action of broadcasting RREQs does not have a big effect on the PDF if the traffic load is light. But as the traffic load increases the RREQ packets will congest the network causing more data packet losses.

Figures 2 through 4 show the variation of packet delivery ratio under different pause time. The node density in each figure is different. With the increase of pause time, mobility is lower, routes are less likely to be broken, and so packet delivery ratio goes up. As the figures show, the PDF of AODV and ECRP are almost the same. Again this improves that ECRP is as efficient as AODV in discovering and $\mathrm{m}: \ldots \ldots$..............

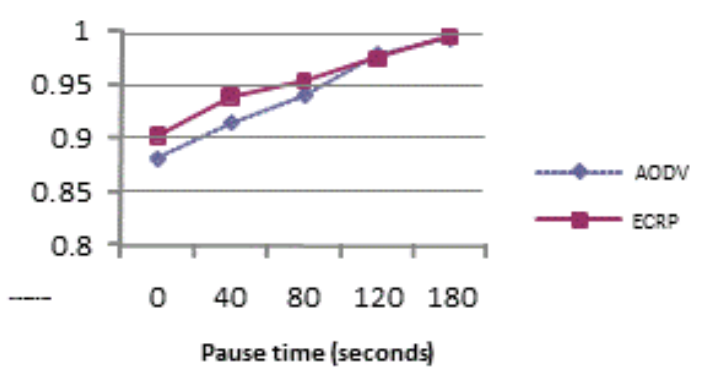

Figure2. Packet delivery Fraction. Number of Nodes: 20 


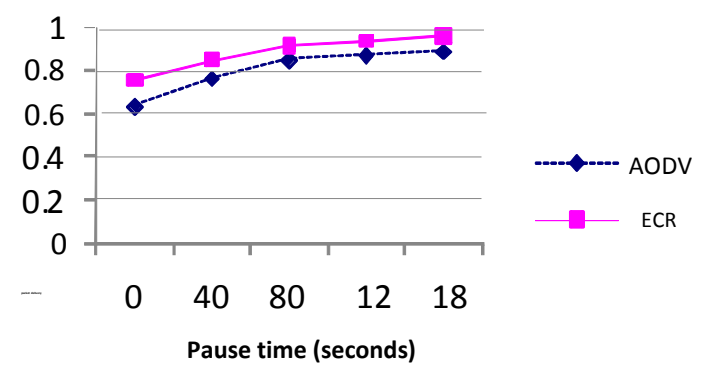

Figure3. Packet Delivery Fraction. Number of Nodes: 40

2) Average-end-to-end Delay (AEED): this is the time the data packets are generated by source to the time at which they are received by the destination. It consists of all possible delays, such as the buffering delay experienced by the data packets during the route discovery phase, queuing delay at the interface queues, retransmission delays at the MAC layer, processing delay at each node, propagation and transfer times, and the length of the paths taken. This metric is in favor of lower delivery rate protocols since the value is only calculated over received data packets, which in general go through shorter path and has less delay. Figure3 shows the AEED for a system with node number 20. As the Figure shows, at high mobility the AEED of ECRP is higher than AODV. In this case external routes tend to break more frequently, which means more broadcasting of control messages. And since these messages have to pass only through bases and backup nodes, then the traffic will suffer from more queuing delays. Also since data has to go through certain nodes, their routes may be longer and cause a higher delay time. As the pause time increases, the routs are maintained and less control messages are transmitted. In this case we see that AODV and ECRP almost have the same AEED.

Figure 4 shows that as the traffic load increases the AEED of AODV and ECRP increases. ECRP still has higher AEED but the difference between it and that of AODV is less. The reason for this is due to the excess in data control packets in AODV. These packets cause the interface queues to be longer and consequently the delay experienced by the data packets will be higher.

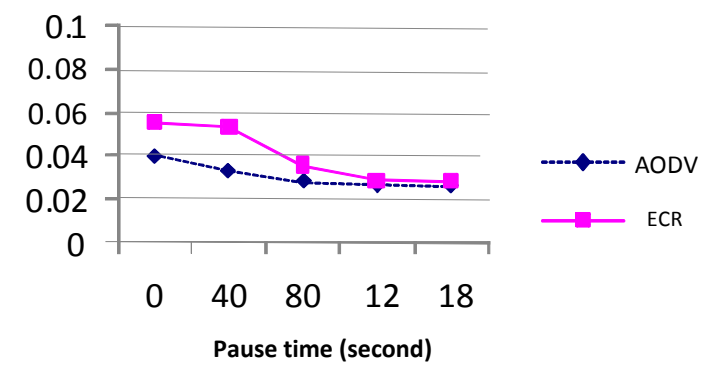

Figure4. Average End-to-End Delay. Number of Nodes: 20 
International Journal of Computer Networks \& Communications (IJCNC) Vol.5, No.3, May 2013

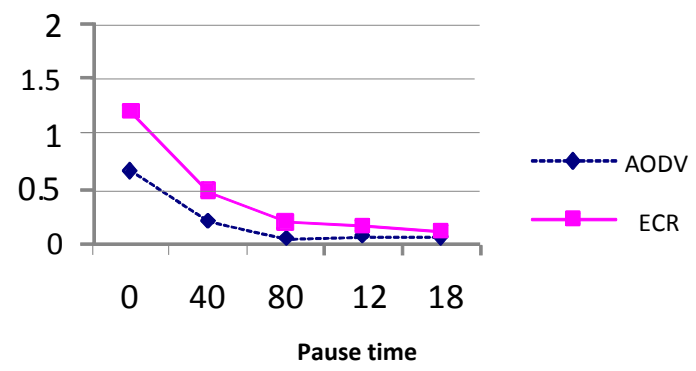

Figure5. Average End-to-End Delay. Number of Nodes: 40

3) Control Routing load (CRL): this is the ratio of the routing messages transmitted in the network to the number of data packets received by the destinations. It measures the efficiency of a routing protocol. The routing messages include all the control messages to establish a route, such as route requests and route replies. For a routing packet transmitted over multiple hops, each hop is considered as one transmission.

Figure 5 shows the CRL for AODV and ECRP in light traffic. At low pause times, ECRP outperforms AODV. This is due to the increase in the number of broken routes and the extra transmission of Control messages used by AODV. Figure6 shows that ECRP outperforms AODV in high traffic system again. When pause time increases, the routing overhead goes down, since mobility is lower and less routes are broken. In addition, ECRP does not flood the RREQs to the whole network, instead only the bases and gateways are concerned.

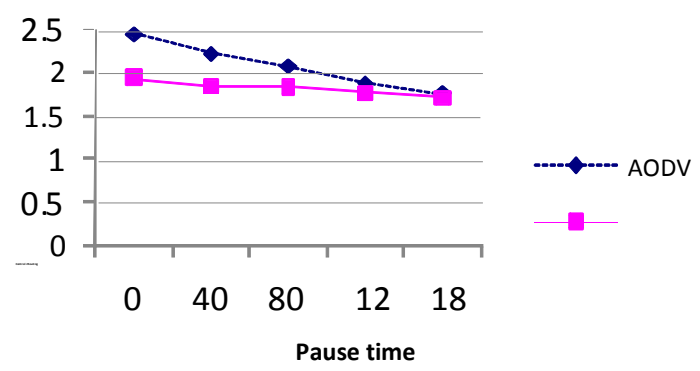

Figure 5. Control Routing. Number of Nodes: 20

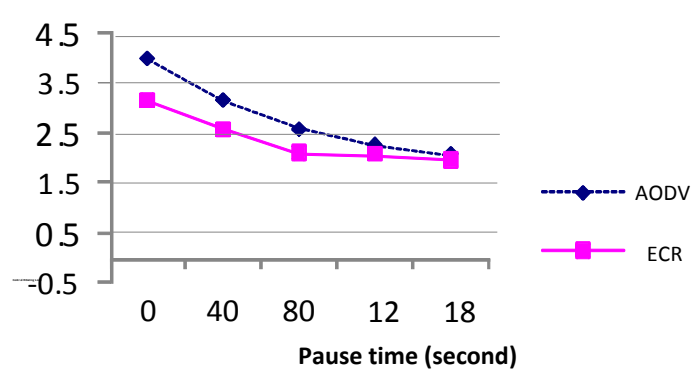

Figure 6. Control Routing. Number of Nodes: 40 
International Journal of Computer Networks \& Communications (IJCNC) Vol.5, No.3, May 2013

\section{Conclusion}

In this paper, I present a scalable routing protocol called ECRP. It is a hybrid scalable routing protocol. The protocol operates by combining the proactive and reactive strategies in routing. In this way, the protocol benefits from the advantages of both approaches. I have evaluated and analyzed the performance of ECRP with ns2 simulator. The protocol was compared against AODV. To evaluate the scalability of protocol the traffic load was increased. The results show that when compared with AODV, ECRP has higher packet delivery fraction and lower routing overhead. The average end-to-end of ECRP is higher than that of AODV. The reason for this is that all paths taken by packets have to pass by bases and backup nodes only. These paths may be longer than the ones taken in AODV routing. In addition, since all traffic passes by bases, the interface queues in these nodes will be longer which will impose more delay.

\section{REFERENCES}

[1] C.E. Perkin (2001) Ad Hoc Networking. Addison-Wesley Longman Publishing Co.

[2] Verotiana H. Rabarijaona, Akeo Masuda, Dinh Chi Hieu, Thomas Bourgeois and Shigeru Shimamoto,(2012) "Cooperative Rate Adaption Scheme For Wireless Networks With Improved Fairness, Delay And Transmission Reliability”, International Journal of Computer Networks \& Communications (IJCNC), Vol.4, No.1, pp 13-26.

[3] Emi Mathews and Ciby Mathew, (2012 ) "Deployment Of Mobile Routers Ensuring Coverage And Connectivity", International Journal of Computer Networks \& Communications (IJCNC), Vol.4, No.1, pp 175-191.

[4] Z. J. Haas, M. R. Pearlman, and P. Samar, (2002) "The Zone Routing Protocol (ZRP) for Ad Hoc Networks", IETF Internet Draft, draftietf- manet-aodv-10.txt.

[5] Elizabeth M. Belding-Royer, (2002) "Hierarchical Routing in Ad hoc Mobile Networks", Wireless Communications and Mobile Computing, VOL 2, NO 7,pp 515-532.

[6] T. Camp; J. Boleng, and V. Davies, (2002) "A survey of mobility models for ad hoc network research", Wireless Communications \& Mobile Computing (WCMC): Special issue on Mobile Ad Hoc Networking: Research, Trends and Applications, No 2: pp 483-502.

[7] E. D. Kaplan, (1996) Understanding GPS-Principles and Applications, Artech House Publisher.

[8] M. Mauve, J. Widmer, and H. Hartenstein, (2001) "A survey on position-based routing in mobile Ad Hoc Networks”, IEEE Network, vol. 15, Issue: 6, pp. 30-39.

[9] J. Li, J. Jannotti, D. S. J. D. Couto, D. R. Karger, and R. Morris, (2000) “A scalable location service for geographic ad hoc routing in MobiCom", Proceedings of the 6th annual international conference on Mobile computing and networking, pp. 120-130.

[10] C. E. Perkins and E. Royer, (1999) "Ad-hoc on-demand distance vector routing," in Proc. of the 2nd IEEE Workshop on Mobile Computing Systems and Applications, pp. 90-100.

[11] A. Hamidian. (2003) “A study of internet connectivity for mobile ad hoc networks in ns 2", Master's thesis, Department of Communication Systems, Lund Institute of Technology, Lund University, Sweden.

[12] K. Fall and K. Varadhan, (2003) "ns notes and documentation," available from http://www.isi.edu/nsnam/ns/.

[13] "IEEE Wireless LAN Medium Access Control (MAC) and Physical Layer (PHY) Specifications," IEEE Std. 802.11-1997.

\section{Author}

Buthayna Al-Sharaa received her B.S degree in Electrical Engineering from Jordan University of Science and Technology/ Jordan in 1997, the M.S in Computer Engineering from Jordan University of Science and Technology in 2006. She is currently an instructor at Al-Balqa Applied University/Jordan. Her research interests include: wireless communication, Performance evaluation of fault tolerant parallel and network systems, algorithms, distributed systems. 